\title{
Ellagitannins from Raspberry (Rubus idaeus L.) Fruit as Natural Inhibitors of Geotrichum candidum
}

\author{
Elżbieta Klewicka ${ }^{1, *}$, Michał Sójka ${ }^{2, *}$, Robert Klewicki ${ }^{2}$, Krzysztof Kołodziejczyk ${ }^{2}$, \\ Lidia Lipińska $^{1}$ and Adriana Nowak ${ }^{1}$ \\ 1 Institute of Fermentation Technology and Microbiology, Lodz University of Technology, \\ Wolczanska 171/173, Lodz 90-924, Poland; lidia.lipinska@dokt.p.lodz.pl (L.L.); \\ adriana.nowak@p.lodz.pl (A.N.) \\ 2 Institute of Food Technology and Analysis, Lodz University of Technology, Stefanowskiego 4/10, \\ Lodz 90-924, Poland; robert.klewicki@p.lodz.pl (R.K.); krzysztof.kolodziejczyk@p.lodz.pl (K.K.) \\ * Correspondence: elzbieta.klewicka@p.lodz.pl (E.K.); michal.sojka@p.lodz.pl (M.S.); \\ Tel./Fax: +48-42-636-59-76 (E.K.); +48-42-636-74-88 (M.S.)
}

Academic Editor: Isabel C. F. R. Ferreira

Received: 30 May 2016; Accepted: 8 July 2016; Published: 13 July 2016

\begin{abstract}
The paper presents the chemical characteristics of ellagitannins isolated from raspberry (Rubus idaeus L.) fruit and their in vitro and in situ antifungal activity against Geotrichum candidum ŁOCK 0511. The study investigated a complex preparation containing various raspberry ellagitannins at a concentration of $86 \% w / w$, as well as pure lambertianin $C$ and sanguiin H-6. The ellagitannin preparation was obtained by extracting raspberry press cake and purifying the extract using Amberlite XAD resin, while individual compounds were isolated by means of preparative HPLC. The complex preparation was analyzed for the content of ellagitannins, anthocyanins, and flavan-3-ols using HPLC and LC-MS. The antifungal activity of the complex ellagitannin preparation and the isolated ellagitannins was determined for the strain Geotrichum candidum. The MIC and MFC values $(10.0 \mathrm{mg} / \mathrm{mL}$ and $30.0 \mathrm{mg} / \mathrm{mL}$, respectively) were found to be the same for lambertianin C, sanguiin $\mathrm{H}-6$, and the complex ellagitannin preparation. The fungistatic activity of the studied ellagitannin preparation at a concentration of $10 \mathrm{mg} / \mathrm{mL}$, as determined by the poisoned medium method, was $65.2 \%$ following 6 day incubation of Geotrichum candidum, with the linear growth rate of only $16.2 \mathrm{~mm} /$ day. The corresponding parameters for the control sample were $0 \%$ and $56 \mathrm{~mm} /$ day, respectively. The study demonstrated both in vitro and in situ antifungal activity of raspberry ellagitannins against Geotrichum candidum.
\end{abstract}

Keywords: antifungal; ellagitannins; raspberry fruit; Geotrichum candidum

\section{Introduction}

Ellagitannins constitute a complex class of polyphenols characterized by one or more hexahydroxydiphenoyl (HHDP) moieties esterified with a sugar, usually glucose. The HHDP group is biosynthetically formed through intramolecular, oxidative (C-C) coupling of suitably oriented neighboring galloyl residues in galloylglucoses. Ellagitannins exhibit very high structural variability due to the different ways in which HHDP residues can be linked with the glucose moiety, and especially due to their strong tendency to form dimeric and oligomeric forms [1,2]. High ellagitannin content has been reported for strawberries, cranberries, cloudberries, and red raspberries. The main raspberry ellagitannins are sanguiin H-6 $(1871 \mathrm{~g} / \mathrm{mol})$ and lambertianin C $(2805 \mathrm{~g} / \mathrm{mol})$. Both compounds are dimers and trimers of casuarictin $(936 \mathrm{~g} / \mathrm{mol})$ respectively, which are formed by intermolecular C-O bonds between an HHDP group and a galloyl group on a neighboring monomer [1]. The content of these compounds in fresh raspberry fruit, depending on the cultivar, ranges from 360 to $750 \mathrm{mg} / \mathrm{kg}$ for sanguiin H-6 and from 280 to $630 \mathrm{mg} / \mathrm{kg}$ for lambertianin C [3]. Polyphenols, and especially 
ellagitannins and flavonoids, have a considerable antimicrobial potential. Many studies have shown that extracts from pomegranate and other plants rich in ellagitannins exhibit a significant inhibitory effect on Escherichia coli, Salmonella enteritidis, Staphylococcus aureus, Bacillus subtilis, Klebsiella pneumoniae, Pseudomonas aeruginosa, Yersinia enterocolitica, Listeria monocytogenes, and Candida albicans, as well as on molds such as Alternaria alternata, Fusarium oxysporum, and Rhizoctonia solani [4-7].

Geotrichum candidum has been isolated from a variety of environments, including river water, air, food, and animal feed [8]. Being used in the food industry, this fungus has been subjected to numerous biotechnological studies in that area. While in the past G. candidum was usually found safe, with a low pathogenic potential, recently it has been reported to cause an increasing number of fungal infections. G. candidum is an opportunistic microorganism [9]. If present in food, this species may infect cancer patients treated with chemotherapy, HIV-positive individuals, those subjected to long-term antibiotic therapy or using corticosteroids, as well as diabetics $[9,10]$. Furthermore, the problem may be expected to further intensify with the increasing population of patients with immune deficiencies. Therefore, it seems necessary to seek safe antifungal compounds or preparations that could be used as natural preservatives in the food industry. The study presents the chemical characteristics of ellagitannins isolated from raspberry (Rubus idaeus L.) fruit and their in vitro and in situ antifungal activity against Geotrichum candidum.

\section{Results and Discussion}

\subsection{Ellagitannin Identification in REP by LC-MS}

Six ellagitannins were identified in the obtained raspberry extract (Table 1) in the process of gradient elution with polyphenols being purified on XAD 1600 resin. Due to the protocol applied, the extract was free of, amongst others, low molecular weight ellagitannins, ellagic acid and its conjugates, and other polyphenols, such as some anthocyanins and flavan-3-ols. Compounds with retention time of $14.57 \mathrm{~min}$ and $17.72 \mathrm{~min}$ gave a major $[\mathrm{M}-\mathrm{H}]^{-}$ion at $m / z 1567.15$ and a doubly charged ion at $m / z$ 783.07. Fragmentation of the doubly charged ion gave a sequence of singly charged ions characteristic of sanguiin H-10 isomers. However, it should be noted that the first isomer fragmented to $\mathrm{m} / \mathrm{z} 1235$, which was associated with the loss of an HHDP (hexahydroxydiphenic acid) residue as described by Kähkönen et al. [11], while the second isomer fragmented to $m / z 1265$, which was linked to the loss of a galloylated glucose as described by Gasperotti et al. [3]. Despite the same mass, the two compounds have different structures, as reported by Gasperotti et al. [3].

Table 1. LC-MS identification of ellagitannins in the raspberry ellagitannin preparation (REP).

\begin{tabular}{ccccc}
\hline $\begin{array}{c}\mathbf{R}_{\mathbf{t}} \\
(\mathbf{m i n})\end{array}$ & MS Data & $\begin{array}{c}\text { MS/MS } \\
\text { Value }\end{array}$ & MS/MS Data & $\begin{array}{c}\text { Tentative Structural } \\
\text { Assignment }\end{array}$ \\
\hline 14.57 & $\begin{array}{c}{[1567.15]^{-1}} \\
{[783.07]^{-2}}\end{array}$ & 783 & $\begin{array}{c}{[1235.07]^{-1}[935.08]^{-1}[633.07]^{-1}} \\
{[469.01]^{-1}[301.00]^{-1}}\end{array}$ & Sanguiin H-10 isomer \\
\hline 15.13 & $\begin{array}{c}{[1250.60]^{-2}} \\
{[833.40]^{-3}}\end{array}$ & 1250 & $\begin{array}{c}{[2200.19]^{-1}[1867.14]^{-1}[1567.14]^{-1}[1235.07]^{-1}} \\
{[933.06]^{-2}[633.07]^{-1}[301.00]^{-1}}\end{array}$ & $\begin{array}{c}\text { Lambertianin C without } \\
\text { ellagic moiety }\end{array}$ \\
\hline 17.72 & $\begin{array}{c}{[1567.15]^{-1}} \\
{[783.07]^{-2}}\end{array}$ & 783 & $\begin{array}{c}{[1265.14]^{-1}[1103.09]^{-1}[935.08]^{-1}[933.07]^{-1}} \\
{[633.07]^{-1}[469.01]^{-1}[301.00]^{-1}}\end{array}$ & Sanguiin H-10 isomer \\
\hline 19.25 & $\begin{array}{c}{[1401.01]^{-2}} \\
{[934.07]^{-3}}\end{array}$ & 1401 & $\begin{array}{c}{[1869.14]^{-1}[1567.14]^{-1}[1235.07]^{-1}[935.08]^{-1}} \\
{[633.07]^{-1}[301.00]^{-1}}\end{array}$ & Lambertianin C isomer \\
\hline 19.75 & $\begin{array}{c}{[1401.01]^{-2}} \\
{[934.07]^{-3}}\end{array}$ & 1401 & {$[1869.14]^{-1}[1567.14]^{-1}[1235.07]^{-1}[935.08]^{-1}$} & Lambertianin C (standard) \\
\hline 2633.07$]^{-1}[301.00]^{-1}$ & {$[1567.14]^{-1}[1235.07]^{-1}[935.08]^{-1}$} & Sanguiin H-6 (standard) \\
\hline 20.57 & {$[934.07]^{-2}$} & $934.07]^{-1}[301.00]^{-1}$ & \\
\hline
\end{tabular}

The compound with a retention time of 15.13 was characterized by the presence of a doubly charged ion at $m / z$ 1250, which fragmented to an ion at $m / z 2200$ losing an HHDP residue as well as 
to an ion at $m / z 1867$ losing a galloylated glucose. Based on the obtained fragmentation and literature data [3], this compound was identified as lambertianin $C$ without one ellagic acid residue. Compounds with retention times of 19.25 and 19.75 exhibited the presence of a doubly charged ion at $m / z 1401$, which fragmented to $m / z 1869,1567$, and 1235, indicating the presence of lambertianin $C$.

The occurrence of two forms suggests the presence of isomers of this compound, which were also observed by Hager et al. [12] and McDougall et al. [13]. In our case, the first compound was identified as an isomer of lambertianin $C$ and the other one as the main (also in quantitative terms) lambertianin $C$ found in raspberry fruit. The compound with a retention time of $20.57 \mathrm{~min}$ gave an ion at $\mathrm{m} / z 1869$, which characteristically fragmented to $m / z 1567,1235,935,633$, and 301, indicating the presence of sanguiin H-6. The identification of lambertianin $\mathrm{C}$ and sanguiin H-6 was also confirmed by comparing retention times and MS spectra of the isolated standards. The same fragmentation patterns have been reported by Gasperotti et al. [3], Hager et al. [12], and Mertz et al. [14].

\subsection{Quantitative Composition of Phenolic Compounds in REP}

Table 2 shows the quantitative ellagitannin composition of REP. In general, ellagitannins account for $92 \%$ of total phenolics. The other compounds, that is, flavan-3-ols and anthocyanins constitute $7.4 \%$ and $0.9 \%$ of total phenolics, respectively. The most abundant ellagitannins in REP are lambertianin $\mathrm{C}$ and sanguiin H-6, which collectively account for $93 \%$ of total ellagitannins (Figure 1), with the remaining ones being sanguiin $\mathrm{H}-10$ isomers and lambertianin $\mathrm{C}$ derivatives. Ellagic acid, a product of ellagitannin hydrolysis, was not detected. The results show that the applied method of extraction (with 60\% acetone) and purification (with XAD 1600 resin) leads to a product characterized by a high phenolic content, with more than $86 \%$ being hydrolysable tannins, including ellagitannins.

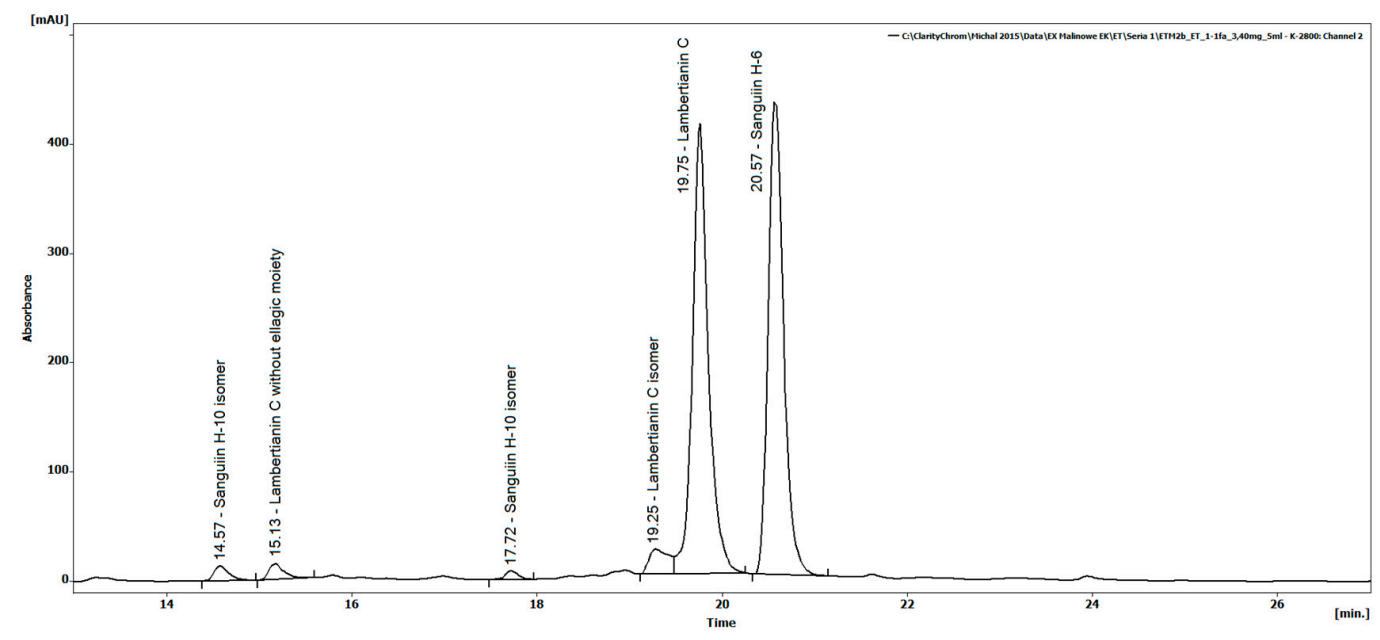

Figure 1. HPLC chromatogram of the raspberry ellagitannin preparation (REP) with UV detection at $250 \mathrm{~nm}$.

Table 2. Ellagitannin (REP), anthocyanin (ACY), and flavanol (FLAVA) content in raspberry ellagitannin extract (REP).

\begin{tabular}{ccc}
\hline Compound & Mean $\mathbf{( m g / \mathbf { 1 0 0 }} \mathbf{g})$ & SD \\
\hline \multicolumn{1}{c}{ Ellagitannins } & & \\
\hline Sanguiin H-10 isomer 1 & 1065 & 57 \\
Lambertianin C without ellagic moiety & 1456 & 97 \\
Sanguiin H-10 isomer 2 & 731 & 47 \\
Lambertianin C isomer & 2961 & 332 \\
Lambertianin C & 44156 & 1705 \\
Sanguiin H-6 & 35917 & 1300 \\
Total ellagitannins & 86287 & 3537 \\
\hline
\end{tabular}


Table 2. Cont.

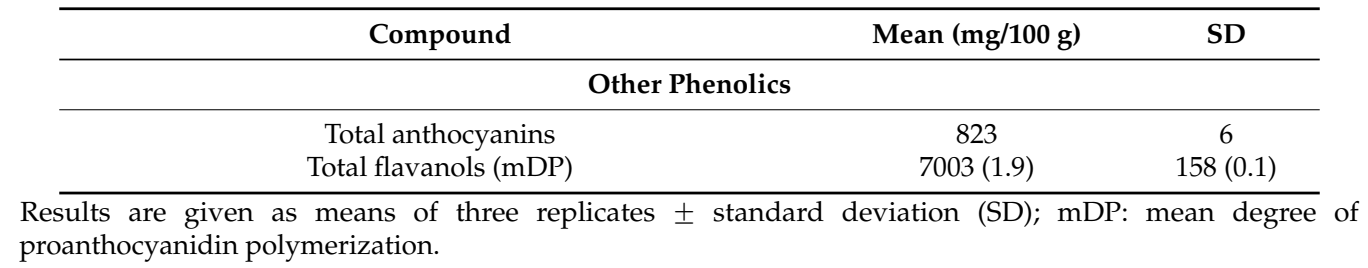

\subsection{Inhibition of Geotrichum Candidum Growth by Ellagitannins}

The strain Geotrichum candidum ŁOCK 0511 used in the presented study, had been isolated as a contaminant of non-pasteurized milk. In the ellagitannin antagonism test, this strain exhibited an inhibition zone of $10.3 \pm 0.47 \mathrm{~mm}$ for REP and of $9.3 \pm 0.47 \mathrm{~mm}$ and $8.3 \pm 1.69 \mathrm{~mm}$ for LC and SH6, respectively. The MIC and MFC for REP were $10.0 \mathrm{mg} / \mathrm{mL}$ and $30.0 \mathrm{mg} / \mathrm{mL}$, respectively, and were identical as those for the LC and SH6 fractions. REP, which is a complex preparation containing both lambertianin C (44.156 g/100 g) and sanguiin H-6 (35.917 g/100 g) (Table 3) exhibits similar antagonistic activity to the pure compounds isolated in this study (Table 3). In respect of chemical structure, sanguin H-6 is a dimer, while lamberitanin $\mathrm{C}$ is a trimer of casuarictin. Hence, taking into consideration their molar masses, in the MIC analysis we introduce the same mass but different amount of moles for both compounds. At the same time, the number of functional groups involved in interactions remains the same. Our research has not identified any link between the degree of polymerization or synergism of both compounds and the MIC parameter. This resulted in the same MIC parameter for the REP preparation, which included $80 \%$ of these compounds in total. It should also be noted that REP preparation also contained $7 \%$ of the flavanols, which in terms of the MIC parameter may compensate for lower ellagitannin content. Foss et al. [15] observed a similar effect, they concluded that crude extract from pomegranate and isolated compound punicalagin showed about the same value of MIC. In this case, punicalagin was the main substance of the crude extract. Moreover, application of the REP preparation in food or pharmaceutical industry for production of an extract rich in ellagitannins would be technologically easier and significantly less expensive than isolation of pure compounds. Therefore, in further study we used REP rather than individual ellagitannin fractions.

Table 3. Minimal inhibitory concentration (MIC), minimal fungicidal concentration (MFC), and antagonistic activity of lambertianin C (LC), sanguiin H-6 (SH6), and the raspberry ellagitannin preparation (REP) with respect to Geotrichum candidum LOCK 0511.

\begin{tabular}{cccc}
\hline Test & REP & LC & SH6 \\
\hline MIC $(\mathrm{mg} / \mathrm{mL})$ & 10.0 & 10.0 & 10.0 \\
MFC $(\mathrm{mg} / \mathrm{mL})$ & 30.0 & 30.0 & 30.0 \\
Zone of growth & $10.3 \pm 0.47^{\text {a }}$ & $9.3 \pm 0.47^{\text {a }}$ & $8.3 \pm 1.69^{\text {a }}$ \\
inhibition $(\mathrm{mm}) \pm$ SD & &
\end{tabular}

SD: standard deviation, ${ }^{\text {a }}$ no statistically significant differences were found in analysis of variance (ANOVA) with the Bonferroni post hoc test $(p \geqslant 0.05)$.

The antifungal activity of extracts from Rubia tinctorum, Carthamus tinctorius and Juglans regia against Geotrichum candidum was studied by Mehrabian et al. [16], who reported effective inhibition of fungal growth by aqueous and methanol extracts from Carthamus tinctorius $(40 \mathrm{~mm}$ and $20 \mathrm{~mm}$, respectively). However, Mehrabian et al. [16] did not specify the chemical characteristics of the plant extracts. In turn, Talibi et al. [17] studied the antagonistic activity of 43 preparations obtained from 36 plant species against Geotrichum candidum. One of the tested substances was a preparation from the leaves and shoots of Rubus ulmifolius Schott, with the MIC and MFC values of $0.3125 \mathrm{mg} / \mathrm{mL}$ and $>5 \mathrm{mg} / \mathrm{mL}$, respectively. Foss et al. [15] studied antifungal activity of crude extract 
and punicalagin from pomegranate against Trichophyton sp. and Microsporium sp. which are human dermatophytes. The authors determine the MIC values of crude extract of pomegranate for these dermatophytes are respectively $125 \mu \mathrm{g} / \mathrm{mL}$ and $250 \mu \mathrm{g} / \mathrm{mL}$. Research conducted by Webster et al. [18] specified the antagonistic activity of water-based plant extracts (Heracleum maximum, Aralia nudicaulis, Glycyrrhiza lepidota, Fragaria virginiana, Populus tremuloides, Artemisia frigida, Artemisia campestris, Solidago gigantea, Alnus viridis, Potentilla simplex, Juniperus communis, Epilobium angustifolium, Betula alleghaniensis, Acorus calamus) against 23 human fungal pathogens belonging to the genera: Candida, Saccharomyces, Cryptococcus, Trichophyton, Microsporum, Epidermophyton, Aspergillus, Fusarium, Rhizopus. Researchers proved that the antifungal activity of plant extracts is individuated and it depends on the extract and the test strains. For instance, for the Fragaria virginiana extract, the MIC parameters were from 400mg/L in case of Trichophyton mentagrophytes, Microsporum canis, Epidermophyton floccosum, Aspergillus fumigatus to $50000 \mathrm{mg} / \mathrm{L}$ for Aspergillus flavus, Fusarium saloni strains. In the analysis provided by Thirach et al. [19], the antifungal activity of an ethanol extract of Acorus calamus was assessed against 28 clinical isolates of Candida sp. using a broth microdilution technique. The MIC was identified as $28,800 \pm 16,320 \mathrm{mg} / \mathrm{L}$, and Acorus calamus was interpreted in this setting as being a potential fungistatic agent.

\subsection{The Fungistatic Activity (FA) of Ellagitannins and the Linear Growth Rate (T) of Filamentous Fungi in Their Presence}

The fungistatic activity (FA) of ellagitannins (REP) and the linear growth rate (T) of the strain Geotrichum candidum ŁOCK 0511 in their presence was determined (Table 4). In the experiments, REP concentrations were selected based on prior studies concerning MIC and MFC.

Table 4. Fungistatic activity (FA) of the raspberry ellagitannin preparation (REP) and the linear growth rate (T) for Geotrichum candidum.

\begin{tabular}{|c|c|c|c|c|c|c|}
\hline \multirow{3}{*}{$\begin{array}{l}\text { Ellagitannins } \\
\quad(\mathrm{mg} / \mathrm{mL})\end{array}$} & \multirow{2}{*}{\multicolumn{4}{|c|}{$\begin{array}{c}\text { FA ( } \%) \\
\text { Time (days) }\end{array}$}} & \multirow{3}{*}{$\begin{array}{c}\text { Growth } \\
\text { Rate } \\
\mathrm{T}_{\text {Ellagitannins }} \\
\text { (mm/day) }\end{array}$} & \multirow{3}{*}{$\begin{array}{c}\text { Growth } \\
\text { Rate } \\
\mathrm{T}_{\text {Control }} \\
\text { (mm/day) }\end{array}$} \\
\hline & & & & & & \\
\hline & 1 & 2 & 3 & 6 & & \\
\hline 1.0 & 24.7 & 27.5 & 16.7 & 13.8 & $51.9 *$ & 61.5 \\
\hline 5.0 & 54.5 & 49.4 & 50.3 & 51.4 & $33.9 * *$ & 56.0 \\
\hline 10.0 & 66.4 & 60.1 & 74.4 & 65.2 & $16.2^{* * *}$ & 56.0 \\
\hline
\end{tabular}

* statistically significant difference $\mathrm{T}_{\text {Ellagitannins }}$ versus $\mathrm{T}_{\text {Control }}\left(p=1.28 \times 10^{-6}\right)$. ${ }^{* *}$ statistically significant difference $\mathrm{T}_{\text {Ellagitannins }}$ versus $\mathrm{T}_{\text {Control }}\left(p=3.09 \times 10^{-4}\right) .{ }^{* * *}$ statistically significant difference $\mathrm{T}_{\text {Ellagitannins }}$ versus $\mathrm{T}_{\text {Control }}\left(p=1.49 \times 10^{-7}\right)$.

The FA of the raspberry ellagitannin preparation against G. candidum increased with ellagitannin concentration in the growth medium. A REP concentration of $10 \mathrm{mg} / \mathrm{mL}$ led to FA of $66.4 \%$ after $24 \mathrm{~h}$ of incubation, which was maintained on day 6 . An ellagitannin concentration lower than MIC $(1.0 \mathrm{mg} / \mathrm{mL})$ resulted in FA of $24.7 \%$ after $24 \mathrm{~h}$ of incubation and $13.8 \%$ on day 6 . In the study of Tabei et al. [17], the FA of a preparation from Rubus ulmifolius Schott at a concentration of $5 \mathrm{mg} / \mathrm{mL}$ was $15 \%$. The studied preparation (from Rubus idaeus L.) at a concentration of $5 \mathrm{mg} / \mathrm{mL}$ exhibited FA ranging from $49.4 \%$ to $54.5 \%$, depending on incubation time (Table 4 ). The linear growth rate (T) for Geotrichum candidum ŁOCK 0511 ranged from $51.9 \mathrm{~mm} /$ day for an ellagitannin concentration of $1.0 \mathrm{mg} / \mathrm{mL}$ to $16.2 \mathrm{~mm} /$ day for $10.0 \mathrm{mg} / \mathrm{mL}$. In contrast, in the control samples $\mathrm{T}$ was from $56.0 \mathrm{~mm} /$ day to $61.5 \mathrm{~mm} /$ day (Table 4). The T values obtained for G. candidum indicate effective fungal inhibition and are statistically significant with respect to the control sample.

\subsection{In Situ Effect of Ellagitannin Preparation}

Hydrolysable tannins are known to effectively inhibit the growth of Gram-negative bacteria, such as Salmonella sp., E. coli, Helicobacter pylori, Campylobacter sp. and Gram-positive bacteria: 
Staphylococcus sp., Clostridium sp., and Bacillus sp. [20]. In contrast, they do not inhibit Gram-positive bacteria, such as Lactobacillus sp., or do so only to a limited extent [21-23]. This gives the opportunity to use ellagitannin preparations in functional foods, especially those containing probiotics. This fact was at the core of the design of in situ tests for the inhibition of a food-contaminating fungus. The selected matrix was milk fermented by the probiotic strain Lactobacillus acidophilus ŁOCK 0842 . The contaminant, Geotrichum candidum, was a pleomorphic filamentous fungus, often termed yeast-like [24]. Experiments were conducted in two variants: the ellagitannin preparation (REP) was added either prior to lactic fermentation (FMREP) or after it (FM + REP). During storage, the control exhibited the presence of Geotrichum candidum ŁOCK 0511 at a level of $5 \log$ units (LU) throughout the storage period (Figure 2). In the FMREP sample, G. candidum counts decreased by 2 LU (to approx. 3 LU) on day 5 of storage and no live cells of this fungus were detected on day 15. In the FM + REP sample, G. candidum counts declined to $1.5 \mathrm{LU}$ on day 5 of storage and no live fungal cells were detected on day 15 , similarly to the FMREP sample.

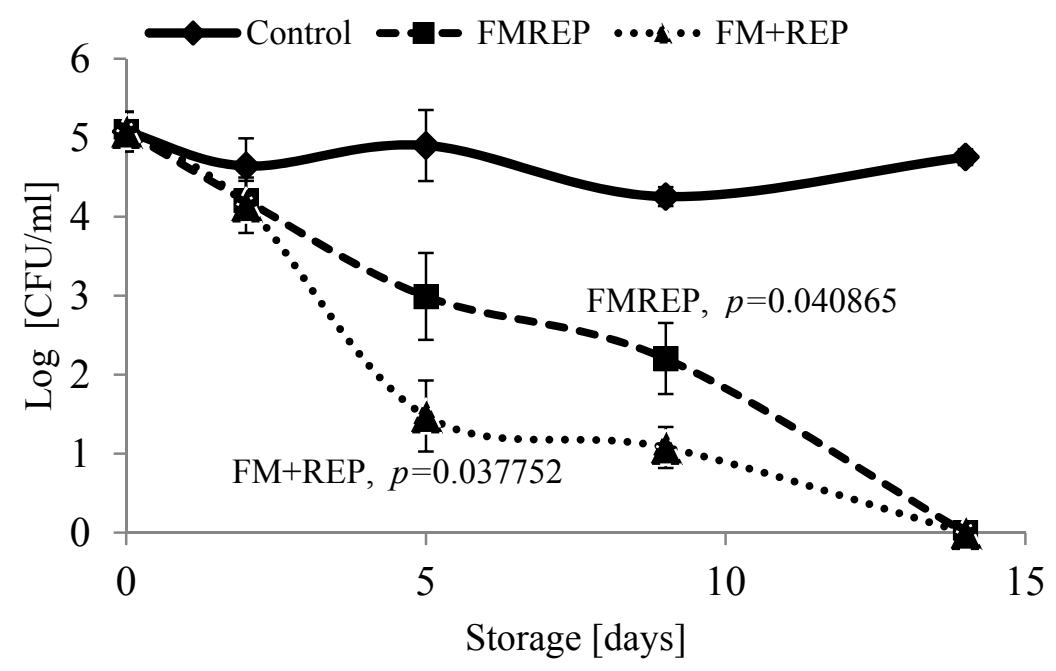

Figure 2. Effect of ellagitannins (10 mg/mL REP) on Geotrichum candidum viability in fermented milk during refrigerated storage $\left(4^{\circ} \mathrm{C}\right)$. FMREP: milk fermented with ellagitannins, FM + REP: ellagitannins added after completed fermentation.

Both samples supplemented with ellagitannins (FMREP and FM + REP) exhibited the same effect, that is, the absence of live G. candidum cells in $1 \mathrm{~mL}$ of fermented milk after 15 days of refrigerated storage. Nevertheless, in the FM + REP sample, to which the ellagitannin preparation was added following fermentation, G. candidum counts were lower by $1.5 \mathrm{LU}$ than in the FMREP sample as early as on day 5 of storage. In the case of FMREP, the ellagitannin preparation was added before the process of milk fermentation. Lactobacills acidophilus bacteria were involved in the milk fermentation process, which may have provoked partial hydrolysis of the compounds. Osawa et al. [25] showed the presence of tannase and gallate decarboxylase in strains of Lactobacillus species isolated from humam feces and fermented food. Ellagitannins under the influence of tannase are hydrolyzed into hexahydroxydiphenic acid, which later on is developed into ellagic acid [26]. Thus, changes in the formula of ellagitannins which may occur during the milk fermentation are likely to influence the dynamics of the antagonistic activity against G. candidum in the FMREP test between the 5th and 10th day of the process.

\subsection{Evaluation of Macroscopic and Microscopic Morphology of Geotrichum Candidum ŁOCK 0511}

Macroscopically, the growth of G. candidum in the presence of $1.0 \mathrm{mg} / \mathrm{mL}$ REP in the medium did not differ from that of the control sample (Figure 3B). In both cases, the colonies were white and velvety. Differences appeared upon the supplementation of the medium with $5.0 \mathrm{mg} / \mathrm{mL}$ and $10 \mathrm{mg} / \mathrm{mL}$ of 
REP. At $5 \mathrm{mg} / \mathrm{mL}$ REP in the medium, the fungal colonies were still white, but lost their smooth and velvety surface structure; they became wavy with a spherical pattern. The growth of G. candidum in the presence $10 \mathrm{mg} / \mathrm{mL}$ REP (Figure 3D) was significantly different from that in the control sample. The fungal colonies were yellowish without a velvety luster and had a wavy surface.
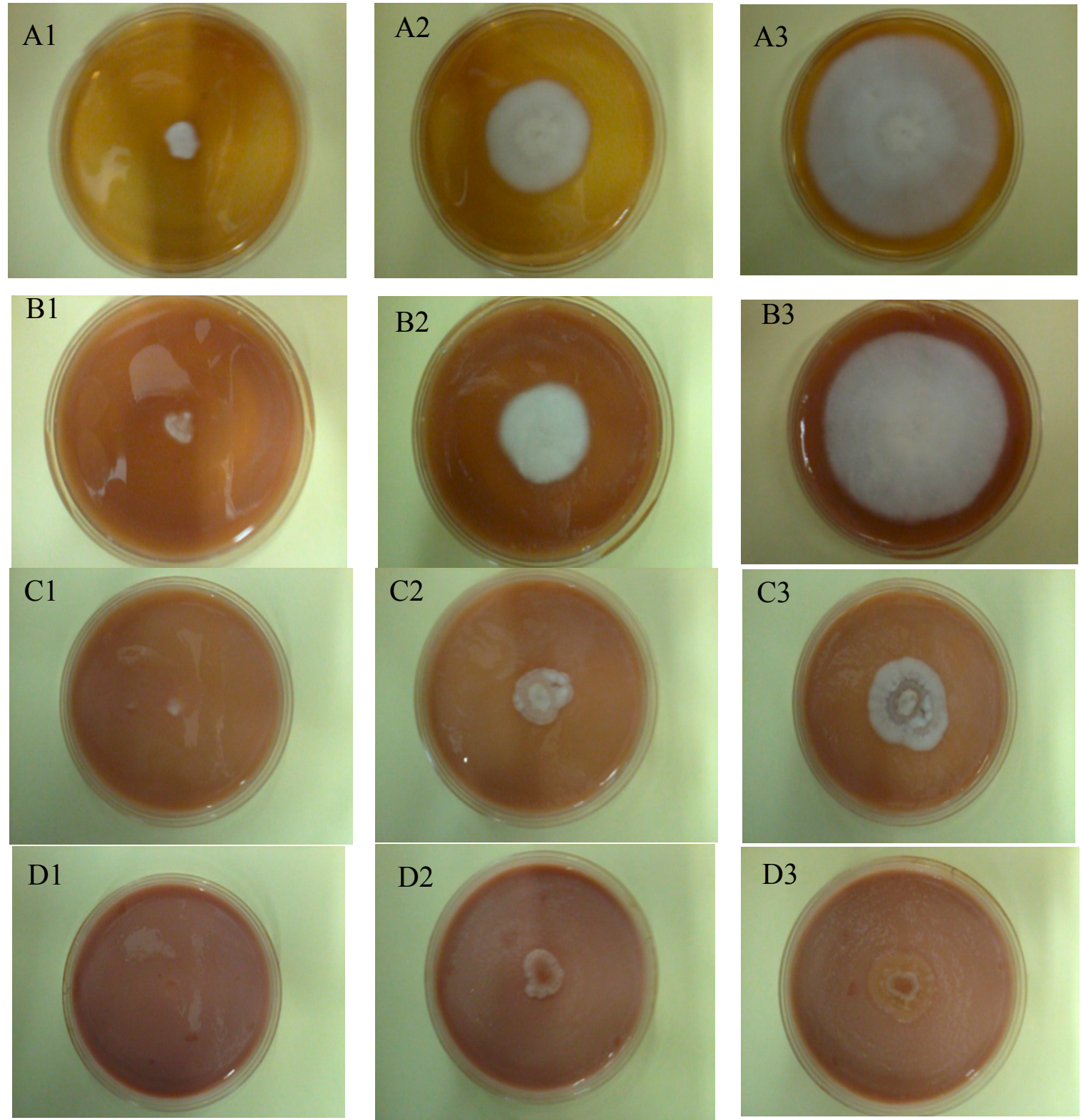

Figure 3. Geotrichum candidum growth on a solid medium supplemented with ellagitannins (REP) (A) Control: 1: after 1 day of incubation, 2: after 3 days of incubation, 3: after 6 days of incubation; (B) $1.0 \mathrm{mg} / \mathrm{mL}$ REP: 1: after 1 day of incubation, 2: after 3 days of incubation, 3: after 6 days of incubation; (C) $5.0 \mathrm{mg} / \mathrm{mL}$ REP: 1: after 1 day of incubation, 2: after 3 days of incubation, 3: after 6 days of incubation; (D) $10.0 \mathrm{mg} / \mathrm{mL}$ REP: 1: after 1 day of incubation, 2: after 3 days of incubation, 3: after 6 days of incubation.

Microscopic slides with living G. candidum cells cultured in the presence of $5 \mathrm{mg} / \mathrm{mL}$ and $10 \mathrm{mg} / \mathrm{mL}$ REP were made (see Figure 3). In the control sample, the hyphae were straight with rounded tips, septa were visible, and arthrospores were present (Figure $4 \mathrm{~A}-\mathrm{a}$ ). The microscopic slide prepared from G. candidum cultured on a medium containing $5.0 \mathrm{mg} / \mathrm{mL}$ REP did not reveal 
arthrospores, and the hyphae were contorted with rounded tips. Furthermore, the hyphae were deformed due to dehydration (Figure 4B-b). Finally, microscopic examination of the G. candidum culture supplemented with $10.0 \mathrm{mg} / \mathrm{mL}$ REP revealed substantial in the morphology of the fungus. The hyphal tips were forked (Figure 4C-c), in contrast to the controls. Cell lysis was observed and no arthrospores were detected. As can be seen, ellagitannin preparation obtained from red raspberry fruit prevented spore formation in G. candidum ŁOCK 0511 and altered hyphal morphology leading to cell wall disorganization and leakage of cytoplasmic material from the hyphae.

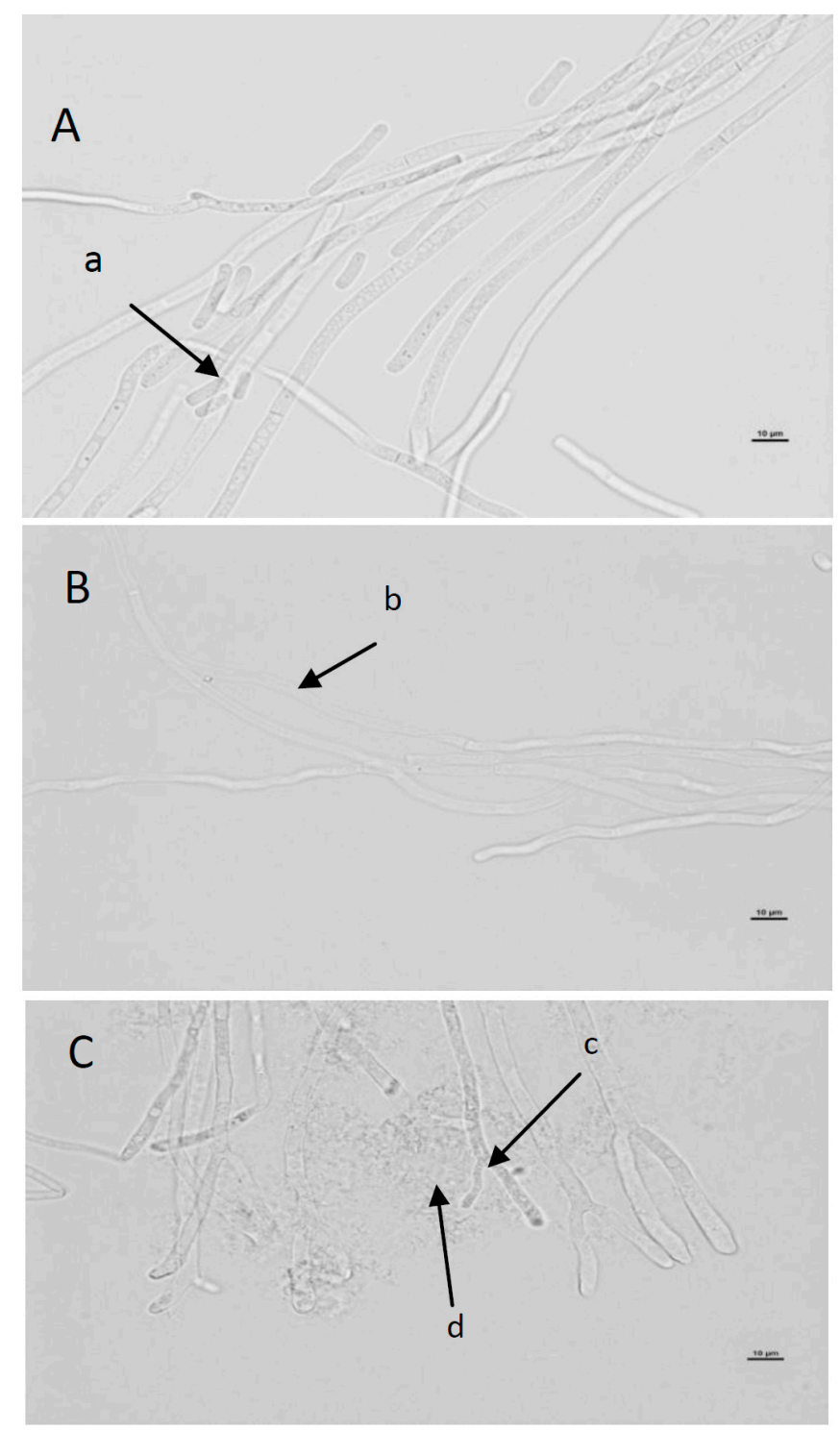

Figure 4. Morphology of the hyphae of Geotrichum candidum cultured in the presence of ellagitannins (REP) on day 6. (A) control, (B) $5.0 \mathrm{mg} / \mathrm{mL} \mathrm{REP,} \mathrm{(C)} 10.0 \mathrm{mg} / \mathrm{mL}$ REP. a: arthrospores, b: dehydrated hyphae, c: forked tips, d: hyphal lysis. Scale bar is $10 \mu \mathrm{m}$.

Similar results were reported by Gatto et al. [27], who studied the antifungal activity of extracts from polyphenol-rich wild herbs against fungi causing fruit and vegetable spoilage: $B$. cinerea (grey mold), M. laxa (inducing brown rot of stone fruits) P. digitatum (Pers.) Sacc. (green mold affecting citrus fruits); P. italicum Wehmer (blue mold affecting citrus fruits), P. expansum Link. (blue mold affecting apples), A. carbonarius (Bainier) Thom, and A. niger. Gatto et al. [27] also observed changes in hyphal morphology, inhibition of spore formation and cell lysis. 
This behavior suggests progressive poisoning of the germ tube caused by some "toxic" compounds in the extract. The greater effect of the ellagitannin extracts on the germ tube elongation rather than on arthrospore germination is probably due to the different composition of the cell wall and to its need to absorb nourishing substances from outside, including also the "toxicants" found in the extract [28]. It can be assumed that the light lipophilic nature of some phenolic compounds contributes to their progressive accumulation in the cell membrane of the fungal pathogens, thus altering its permeability and affecting some transport mechanisms [27,29].

\section{Materials and Methods}

\subsection{Plant Material}

Frozen raspberry fruits of the Polka cultivar collected in August 2013 from plantations in Lublin Province were purchased from the company Cajdex (Łódź, Poland). The fruits were stored in polyethylene bags at $-18^{\circ} \mathrm{C}$ prior to ellagitannin extraction.

\subsection{Fruit Processing and Ellagitannin Extraction}

Ellagitannins were extracted from raspberry press cake, following juice pressing from fruit pulp. Prior to processing, $30 \mathrm{~kg}$ of fruits was defrosted and ground using a fruit grinder (Model 886.9, Zelmer, Rzeszów, Poland). The pulp was heated to $50{ }^{\circ} \mathrm{C}$ and treated with the enzymatic preparation Rohapect $10 \mathrm{~L}$ (AB Enzymes, Darmstadt, Germany) at a dose of $0.2 \mathrm{~mL}$ per $1 \mathrm{~kg}$ of pulp. Enzyme treatment lasted for $1 \mathrm{~h}$ at $50^{\circ} \mathrm{C}$, with the pulp stirred every $10 \mathrm{~min}$. Subsequently, the pulp was pressed using a laboratory basket press (self-manufactured, Lodz University of Technology, Lodz, Poland) to separate juice from the press cake. The latter was extracted with water ( $1 / 2$ of press cake weight) for $15 \mathrm{~min}$ at $50{ }^{\circ} \mathrm{C}$ to remove water-soluble anthocyanins. Next, the press cake was pressed again to obtain secondary juice and press cake $(3.4 \mathrm{~kg})$.

The press cake was extracted in two steps using $60 \%$ acetone at ambient temperature. The mass ratio of the press cake to the extractant was 1:5 and extraction time was $8 \mathrm{~h}$ per one step. The extraction process was augmented with shaking using an orbital shaker (Elmi DOS-10 L, Aizkraukles, Riga, Latvia) at $150 \mathrm{rpm}$. After the first step, raw extract was filtered through cotton filter cloth in order to separate solids, while in the second step, the post-extraction residue was extracted according to the same procedure. The extracts obtained from the two steps were mixed and filtered through a $3.6 \mathrm{~mm}$ thick Hobrafilt S40N cellulose filter with $5 \mu \mathrm{m}$ nominal retention (Hobra-Školnik S.R.O., Broumov, Czech). Acetone was removed from the raw extract using a Basis Hei-VAP HL rotary vacuum evaporator (Heidolph, Schwabach, Germany) at $60{ }^{\circ} \mathrm{C}$ and under reduced pressure of 450-72 mbar. The extract without acetone was again passed through a Hobrafilt $540 \mathrm{~N}$ filter, and then purified on a $90 \mathrm{~cm} \times 1.6 \mathrm{~cm}$ column packed with Amberlite XAD $1600 \mathrm{~N}$ resin (DOW, Midland, MI, USA). The extract was injected at a rate of approx. $15 \mathrm{~mL} / \mathrm{min}$. Elution was carried out at $10 \mathrm{~mL} / \mathrm{min}$ using water/ethanol solutions with ethanol concentrations of $10 \%, 20 \%, 30 \%, 40 \%, 50 \%$, and $60 \%$, consecutively. The volume of solutions with each ethanol concentration equaled the volume of the column. The eluate obtained using the $40 \%$ ethanol solution contained the highest concentrations of lambertianin C and sanguiin H-6. Subsequently, ethanol was removed and the eluate was concentrated to approx. $5^{\circ}$ Brix using a Basis HEI-VAP HL rotary vacuum evaporator at $60{ }^{\circ} \mathrm{C}$ under pressure reduced from 135 to 72 mbar. The concentrated extract was then freeze-dried $\left(-32^{\circ} \mathrm{C}, 48 \mathrm{~h}\right.$; Christ, Alpha 1-2 LDplus, Osterode am Harz, Germany). In the process, $30.8 \mathrm{~g}$ of raspberry ellagitannin preparation (REP) was obtained in the form of a red powder.

\subsection{Isolation of Sanguiin $H-6$ and Lambertianin $C$}

Individual ellagitannins were isolated from an aqueous REP solution with a concentration of $4 \mathrm{mg} / \mathrm{mL}$ using a Knauer preparative HPLC system (Berlin, Germany). The chromatograph was composed of two gradient-forming pumps (Knauer K-501), a Luna 10 u C18 (2) 100A AXIA-packed 
(250 mm $\times 21.2 \mathrm{~mm} ; 10 \mu \mathrm{m}$ ) column (Phenomenex, Torrance, CA, USA), a UV-Vis detector, a Foxy R1 Teledyne ISCO fraction collector (Lincoln, NE, USA), and Eurochrom 2000 chromatographic software. Two eluents were used for separation: eluent $\mathrm{A}-0.1 \%$ formic acid in water, eluent $\mathrm{B}-75 \%$ methanol. The flow rate was $15 \mathrm{~mL} / \mathrm{min}$. The following gradient was used: $0-5 \mathrm{~min} 10 \% \mathrm{~B} ; 5-30 \mathrm{~min} 10 \%-25 \%$ B; 30-50 $\min 25 \%-35 \%$ B; 50-65 $\min 35 \%-40 \%$ B; 65-70 $\min 40 \%-10 \%$ B; and 70-75 $\min 10 \%$ B. The injection volume was $500 \mu \mathrm{L}$. The detection parameter was set to $260 \mathrm{~nm}$. Lambertianin $C$ and sanguiin H-6 peaks were collected from 200 separations. Methanol was removed from the obtained solutions using a Basis HEI-VAP HL rotary vacuum evaporator (Heidolph, Schwabach, Germany) at $60{ }^{\circ} \mathrm{C}$ under a pressure of $100 \mathrm{mbar}$. Then, the solutions were freeze-dried, producing $96.5 \mathrm{mg}$ of sanguiin H-6 (white-beige powder) and $102 \mathrm{mg}$ of lambertianin C (white-creamy powder).

\subsection{Identification of Ellagitannins}

A Dionex Ultimate 3000 high performance liquid chromatograph (HPLC) from Thermo Fisher Scientific (Germering, Germany) coupled with a diode array detector (DAD,) and a Q Exactive Orbitrap mass spectrometer (MS, Thermo Fisher Scientific, Bremen, Germany) were used for the identification of ellagitannins. The solvents used for separations were as follows: solvent $A, 1 \%(v / v)$ formic acid in water and solvent $\mathrm{B}$, an 80:20 $(v / v)$ acetonitrile:water solution. The following gradient was used: 0-6.5 $\min 5 \%(v / v)$ B; 6.5-12.5 min 5\%-15\% (v/v) B; 12.5-44 min 15-45\% (v/v) B; 44-45 min 45\%-75\% $(v / v) \mathrm{B} ; 45-50 \mathrm{~min} 75 \%(v / v) \mathrm{B} ; 50-52 \mathrm{~min} 75 \%-5 \%(v / v) \mathrm{B} ; 52-65 \mathrm{~min} 5 \%(v / v)$ B. A Luna C18 (2) 100A $250 \times 4.6 \mathrm{~mm}$ i.d., $5 \mu \mathrm{m}$ column was used with a Luna C18 $4 \mathrm{~mm} \times 3 \mathrm{~mm}$ pre-column (both from Phenomenex). The column temperature was set to $35^{\circ} \mathrm{C}$, the flow rate was $1 \mathrm{~mL} / \mathrm{min}$, and the injection volume was $20 \mu \mathrm{L}$. Chromatographic data were collected using Xcalibur software (Thermo Fisher Scientific, Waltham, MA, USA). The MS system coupled to the HPLC apparatus was an Orbitrap mass spectrometer equipped with an H-ESI probe used in the negative mode. The source parameters were as follows: vaporizer temperature $500{ }^{\circ} \mathrm{C}$, ion spray voltage $4 \mathrm{kV}$, capillary temperature $400{ }^{\circ} \mathrm{C}$, with sheath gas and auxiliary gas flow rates being 75 and 20 units, respectively. The detector was operated in either the full MS or full MS/dd-MS ${ }^{2}$ scan modes. In the full MS mode, the scan rage of $m / z$ 200-2000 was used. To generate MS² data, the full MS/dd-MS² scan mode was used. In this mode, the selected precursor ions entered into an HDC collision cell, where they were fragmented with normalized collision energy (NCE) to obtain product ion spectra $\left(\mathrm{MS}^{2}\right)$. In our experiments, the NCE used to generate $\mathrm{MS}^{2}$ spectra was set to 20. Tuning and optimization were performed using direct injection of a REP diluted in an 80:20 $(v / v)$ mixture of mobile phases A and B at a flow rate of $0.25 \mathrm{~mL} / \mathrm{min}$. The results of ellagitannin identification are given in Table 2.

\subsection{Quantification of Ellagitannins}

First, $3 \mathrm{mg}$ of REP was dissolved in $5 \mathrm{~mL} \mathrm{50 \%} \mathrm{methanol} \mathrm{and} \mathrm{the} \mathrm{solution} \mathrm{was} \mathrm{sonicated} \mathrm{for} 5 \mathrm{~min}$. Subsequently, the solution was diluted with mobile phase A at a ratio of 1:1 (v/v), centrifuged at $15,000 \times g$ (MPW-260R centrifuge; Med. Instruments, Warsaw, Poland) and transferred into a vial. The content of ellagitannins was determined using a Smartline chromatograph (Knauer, Berlin Germany), composed of a degasser (Manager 5000), two pumps (P1000), an autosampler (3950), a thermostat, and a PDA detector (2800). Ellagitannins were separated on a $250 \mathrm{~mm} \times 4.6 \mathrm{~mm}$ i.d., $5 \mu \mathrm{m}$ Gemini C18 110A column (Phenomenex, Torrance, CA, USA) by gradient elution with $0.05 \%(v / v)$ phosphoric acid:water (solvent $\mathrm{A}$ ) and 83:17 ( $v / v)$ acetonitrile:water with 0.05\% phosphoric acid (solvent $\mathrm{B}$ ). The column temperature was set to $35^{\circ} \mathrm{C}$, the flow rate was $1.25 \mathrm{~mL} / \mathrm{min}$, and the gradient program was as follows: $0-5 \min 5 \%(v / v) \mathrm{B} ; 5-10 \min 5 \%-15 \%(v / v) \mathrm{B} ; 10-35 \mathrm{~min} 15 \%-40 \%(v / v) \mathrm{B} ; 35-40 \mathrm{~min}$ $40 \%-73 \%(v / v) \mathrm{B} ; 40-44 \mathrm{~min} 73 \%(v / v) \mathrm{B} ; 44-46 \mathrm{~min} 73 \%-5 \%(v / v) \mathrm{B} ; 46-54 \mathrm{~min}, 5 \%(v / v) \mathrm{B}$. The injection volume was $20 \mu \mathrm{L}$. Data were collected using ClarityChrom v. 3.0.5.505 software (Knauer). Ellagitannins were detected at $250 \mathrm{~nm}$, and standard curves for lambertianin C and sanguiin H-6 were used for quantification. The sanguiin H-6 curve was used for the quantification of sanguiin H-10 isomers. The lambertianin $\mathrm{C}$ curve was used for the quantification of lambertianin $\mathrm{C}$ derivatives. 


\subsection{HPLC Analysis of Anthocyanins}

Anthocyanins in REP were quantified pursuant to the protocol given in Sójka et al. [30]. The same apparatus and separation conditions were used. A standard curve using an external standard of cyanidin-3-O-glucoside was used.

\subsection{HPLC Analysis of Flavanols (Sum of proAnthocyanidins and Catechins)}

Flavanols were quantified in REP by acid-catalyzed degradation of polymeric proanthocyanidins in excess phloroglucinol, as described in Sójka et al. [31]. The same apparatus and separation conditions were used. Prior to analysis, samples of fruits, juices, and press cake were freeze dried for $48 \mathrm{~h}$ at $-36{ }^{\circ} \mathrm{C}$ (Christ, Alpha 1-2 LDplus, Osterode am Harz, Germany).

\subsection{Microorganisms}

The study involved Geotrichum candidum 0511, a strain of filamentous fungi deposited with the Collection of Industrial Microorganisms of the Institute of Fermentation Technology and Microbiology ŁOCK 105, Lodz University of Technology (Poland). Two morphotypes of Geotrichum candidum have been described in the literature. The first one forms cream-colored yeast-like colonies which produce abundant arthrospores, but grow poorly. The other morphotype forms white felt-like colonies similar to those of molds, consisting of vegetative septate hyphae with few arthrospores, and is capable of fast and abundant growth $[9,10]$. The strain Geotrichum candidum ŁOCK 0511, which was used in this study, belongs to the latter morphotype.

The strain was stored on YEA slants (Yeast Extract Agar, Sigma-Aldrich, Saint Louis, MO, USA) at $4{ }^{\circ} \mathrm{C}$ and activated either on Sabouraud dextrose broth or on agar (Merck KGaA, Darmstadt, Germany), as need be.

\subsection{Antagonistic Activity of Ellagitannins}

Antagonistic activity was assessed by the standard disk diffusion method. Sterile paper discs (5.0 mm diameter, Oxoid, Hampshire, UK) were impregnated with $20 \mu \mathrm{L}$ of ellagitannin preparation (REP), lambertianin C (LC) and Sanguiin H-6 (SH6) at a concentration of $30 \mathrm{mg} / \mathrm{mL}$, and placed on Sabouraud dextrose agar (Merck) plates inoculated with $100 \mu \mathrm{L}$ of fungal suspension adjusted to $10^{6}$ colony forming units (CFU) per mL. Discs containing $50 \mathrm{mg} / \mathrm{mL}$ dimethyl sulfoxide (DMSO, Merck) were used as a negative control. Experiments were done in triplicate and the results were presented as mean inhibition of strain growth (after subtracting disc diameter) \pm standard deviation. One-way analysis of variance (ANOVA) with the Bonferroni post hoc test $(p \leqslant 0.05)$ was applied to determine differences between REP, LC and SH6 samples.

\subsection{Inhibition of Geotrichum Candidum Growth by Ellagitannins}

The antifungal properties, minimal inhibitory concentration (MIC), and minimal fungicidal concentration (MFC) of the ellagitannin preparation (REP), lambertianin C (LC) and Sanguiin H-6 (SH6) were determined by the broth dilution method in test tubes. Ellagitannin solutions were prepared and diluted in DMSO $(50 \mathrm{mg} / \mathrm{mL}$ ) (Merck). Subsequently, $100 \mu \mathrm{L}$ of diluted ellagitannin solutions were added to $4.9 \mathrm{~mL}$ of Sabouraud dextrose broth (Merck) containing a suspension of arthrospores at a concentration of $10^{5}$ spores $/ \mathrm{mL}$, so that the final ellagitannin concentration ranged from $0.05 \mathrm{mg} / \mathrm{mL}$ to $30 \mathrm{mg} / \mathrm{mL}$. The samples were incubated at $30^{\circ} \mathrm{C}$ for $72 \mathrm{~h}$. The minimum inhibitory concentration was defined as the lowest concentration of the compound that inhibited visible growth of the fungus. Subsequently, $1 \mathrm{~mL}$ was collected from the samples with no visible growth and plated on Sabouraud dextrose agar. The samples were incubated at $30^{\circ} \mathrm{C}$ for $48 \mathrm{~h}$. The minimum fungicidal concentration was defined as the lowest concentration of the compound leading to no fungal growth [32]. 


\subsection{Fungistatic Activity and Linear Growth Rate of Filamentous Fungi in the Presence of Ellagitannins}

Ellagitannin-induced inhibition of the linear growth rate of Geotrichum candidum ŁOCK 0511 was studied by means of the poisoned medium method [33]. Sabouraud dextrose agar (Merck) was supplemented with $1.0 \mathrm{mg} / \mathrm{mL}, 5.0 \mathrm{mg} / \mathrm{mL}$, and $10.0 \mathrm{mg} / \mathrm{mL}$ of REP (the amounts specified refer to ellagitannin content). The control was a culture without REP. Tests were done in triplicate. The linear growth rate of Geotrichum candidum $(\mathrm{T})$ was calculated based on fungal growth measurements performed every $24 \mathrm{~h}$ according to the formula:

$$
\mathrm{T}=(\mathrm{A} / \mathrm{D})+\left(\mathrm{b}_{1} / \mathrm{d}_{1}\right)+\ldots+\left(\mathrm{b}_{\mathrm{n}} / \mathrm{d}_{\mathrm{n}}\right)(\mathrm{mm} / 24 \mathrm{~h})
$$

where $\mathrm{T}$-linear growth rate, $\mathrm{A}$ - mean fungal colony diameter in $\mathrm{mm}, \mathrm{D}$ - duration of experiment, $b_{1} \ldots b_{n}$-colony diameter growth in $m m, d_{1} \ldots d_{n}$-number of days since the last measurement.

The fungistatic activity of ellagitannins (FA) against filamentous fungi was determined based on fungal growth inhibition calculated according to the formula:

$$
\mathrm{FA}=((\mathrm{K}-\mathrm{A}) / \mathrm{K}) \times 100(\%)
$$

where: FA-fungistatic activity in $\%, \mathrm{~K}$-mean fungal colony diameter on the control plate, A-mean fungal colony diameter on the plate containing ellagitannins. The experimental data are expressed as mean values. One-way analysis of variance (ANOVA, $p \leqslant 0.05$ ) was applied to find differences between the experimental samples and the control samples.

\subsection{In Situ Effect of the Raspberry Ellagitannin Preparation}

The in situ antagonistic activity of REP against Geotrichum candidum was determined in fermented milk. Reconstructed 10\% milk (skimmed powdered milk, from the company BTL sp. z o.o., Lodz, Poland) was pasteurized at $80{ }^{\circ} \mathrm{C}$ for $10 \mathrm{~min}$. After cooling down, the milk was inoculated with the Lactobacillus acidophilus ŁOCK 0842 strain of lactic acid bacteria (Collection of Industrial Microorganisms of the Institute of Fermentation Technology and Microbiology ŁOCK 105, Lodz University of Technology) in the amount of $1.5 \times 10^{6} \mathrm{CFU} / \mathrm{mL}$ and supplemented with an aqueous solution of the ellagitannin preparation (REP), for ellagitannin concentration in milk to reach $10 \mathrm{mg} / \mathrm{mL}$. Milk prepared in this way (FMREP) was incubated for $48 \mathrm{~h}$ at $37{ }^{\circ} \mathrm{C}$. Alternatively, in some samples REP was added to milk following fermentation, with the other parameters remaining constant $(\mathrm{FM}+\mathrm{REP})$.

Milk samples (FMREP and FM + REP) were inoculated with Geotrichum candidum in the amount of $1.2 \times 10^{5} \mathrm{CFU} / \mathrm{mL}$. The obtained fermented milk, inoculated with the fungus, was stored under refrigeration $\left(4^{\circ} \mathrm{C}\right)$. During storage, G. candidum counts were monitored by the plate method on days $2,5,9$, and 15 . The control sample was fermented milk inoculated with the fungus following lactic fermentation, without REP.

Experiments were conducted in triplicate with the results given as mean values with standard deviations. One-way analysis of variance (ANOVA, $p \leqslant 0.05$ ) was applied to find differences between samples with REP and the control samples.

\subsection{Microscopic Analysis}

Photomicrographs of Geotrichum candidum mycelium were acquired with a Nikon Eclipse Ci H600L (Nikon, Tokyo, Japan) microscope (total magnification $400 \times$ ) operated with NIS-Elements Advanced Research v. 3.0 software (Nikon).

\section{Conclusions}

Given the increasing numbers of immunologically compromised patients, it is of utmost importance to control microbiological contaminants in food. The widely used chemical preservatives 
may give rise to allergic disorders and are not very well accepted by the consumers. Therefore, it is necessary to seek natural biologically active compounds which could be applied as food bio-preservatives. The present study demonstrated that ellagitannins obtained from red raspberry (Rubus idaeus L.) fruit exhibit in vitro and in situ antifungal activity against Geotrichum candidum.

Acknowledgments: This study was supported by a grant from the National Science Center (grant no. 2013/09/B/NZ9/01806).

Author Contributions: Elżbieta Klewicka and Michał Sójka supervised and designed the study, performed the experiments and wrote the manuscript; Robert Klewicki analyzed the data, Krzysztof Kołodziejczyk edited the manuscript, and Lidia Lipińska and Adriana Nowak corrected the manuscript.

Conflicts of Interest: The authors declare no conflict of interest.

\section{References}

1. Yoshida, T.; Amakura, Y.; Yoshimura, M. Structural features and biological properties of ellagitannins in some plant families of the order Myrtales. Int. J. Mol. Sci. 2010, 11, 79-106. [CrossRef] [PubMed]

2. Landete, J.M. Ellagitannins, ellagic acid and their derivative metabolites: A review about source, metabolism, function and health. Food Res. Int. 2011, 44, 1150-1160. [CrossRef]

3. Gasperotti, M.; Masuero, D.; Vrhovsek, U.; Guella, G.; Mattivi, F. Profiling and accurate quantification of Rubus ellagitannins and ellagic acid conjugates using direct UPLC-Q-TOF HDMS and HPLC-DAD analysis. J. Agric. Food Chem. 2010, 58, 4602-4616. [CrossRef] [PubMed]

4. Ascacio-Valdés, J.; Burboa, E.; Aguilera-Carbo, A.F.; Aparicio, M.; Perez-Schmidt, R.; Rodrígez, R.; Aguilar, C.N. Antifungal ellagitannin isolated from Euphorbia antisyphilitia Zucc. Asian Pac. J. Trop. Biomed. 2013, 3, 41-46. [CrossRef]

5. Türkyılmaz, M.; Tağı, S.; Dereli, U.; Özkan, M. Effects of various pressing programs and yields on the antioxidant activity, antimicrobial activity, phenolic content and colour of pomegranate juices. Food Chem. 2013, 138, 1810-1818. [CrossRef] [PubMed]

6. Al-Zoreky, N.S. Antimicrobial activity of pomegranate (Punica granatum L.) fruit peels. Int. J. Food Microbiol. 2009, 134, 244-248. [CrossRef] [PubMed]

7. Navarro, V.; Villarreal, M.L.; Rojas, G.; Lozoya, X. Antimicrobial evaluation of some plants used in Mexican traditional medicine for the treatment of infectious diseases. J. Ethnopharmacol. 1996, 53, 143-147. [CrossRef]

8. O'Brien, M.; O'Keiely, P.; Fuller, H.T. Fungi isolated from contaminated baled grass silage on farms in the Irish Midlands. FEMS Microbiol. Lett. 2005, 247, 131-135. [CrossRef] [PubMed]

9. Skóra, M.; Witalis, J.; Krzysciak, P.; Macura, A.B. Fungal genus Geotrichum: An opportunistic pathogen of humans. Post. Mikrobiol. 2009, 48, 125-132.

10. Pottier, I.; Gente, S.; Vernoux, J.P.; Guéguen, M. Safety assessment of dairy microorganisms: Geotrichum candidum. Int. J. Food Microbiol. 2008, 126, 327-332. [CrossRef] [PubMed]

11. Kähkönen, M.; Kylli, P.; Ollilainen, V.; Salminen, J.-P.; Heinonen, M. Antioxidant Activity of Isolated Ellagitannins from Red Raspberriesand Cloudberries. J. Agric. Food Chem. 2012, 60, 1167-1174. [CrossRef] [PubMed]

12. Hager, T.J.; Howard, L.R.; Liyanage, R.; Lay, J.O.; Prior, R.L. Ellagitannin composition of blackberry by HPLC-ESI-MS and MALDI-TOF-MS. J. Agric. Food Chem. 2008, 56, 661-669. [CrossRef] [PubMed]

13. McDougall, G.; Martinussen, I.; Stewart, D. Towards fruitful metabolomics: High throughput analyses of polyphenol composition in berries using direct infusion mass spectrometry. J. Chromatogr. B 2008, 871, 362-369. [CrossRef] [PubMed]

14. Mertz, C.; Cheynier, V.; Günata, Z.; Brat, P. Analysis of phenolics compounds in two blackberry species (Rubus glaucus and Rubus adenotrichus) by high-performance liquid chromatography with diode array detection and electrospray ion trap mass spectrometry. J. Agric. Food Chem. 2007, 55, 8616-8624. [CrossRef] [PubMed]

15. Foss, R.S.; Nakamura, C.V.; Udea-Nakamura, T.; Cortez, D.A.G.; Endo, E.H.; dias Filho, B.P. Antifungal activity of pomegranate peel extract and isolated compound punicalagin against dermatophytes. Ann. Clin. Microbiol. Antimicrob. 2014, 13, 32. [CrossRef] [PubMed] 
16. Mehrabian, S.; Majd, A.; Majd, I. Antimicrobial effect of three plants (Rubia tinctorum, Carthamus tinctorius and Juglans regia) on some airborne microorganisms. Aerobiologia 2000, 16, 455-458. [CrossRef]

17. Talibi, I.; Askarne, L.; Boubaker, H.; Boudyach, E.H.; Msanda, F.; Saadi, B.; ait ben Aoumar, A. Antifungal activity of some Moroccan plants against Geotrichum candidum the casual agent of postharvest citrus sour rot. Crop Protect. 2012, 35, 41-46. [CrossRef]

18. Webster, D.; Taschereau, P.; Belland, R.J.; Sand, C.; Rennie, R.P. Antifungal activity of medicinal plant extracts; preliminary screening studies. J. Ethnopharmacol. 2008, 115, 140-146. [CrossRef] [PubMed]

19. Thirach, S.; Tragoolpua, K.; Punjaisee, S.; Khamwan, C.; Jatisatienr, C.; Kunyanone, N. Antifungal activity of some medicinal plant extracts against Candida albicans and Cryptococcus neoformans. Acta Hortic. 2003, 597, 217-221. [CrossRef]

20. Daglia, M. Polyphones as antimicrobial agents. Curr. Opin. Biotechnol. 2012, 23, 174-181. [CrossRef] [PubMed]

21. Puupponen-Pimiä, R.; Nohynek, L.; Meier, C.; Heinonen, M.; Hopia, A.; Oksman-Caldentey, K.-M. Antimicrobial properties of phenolic compound from berries. J. Appl. Microbiol. 2001, 90, 494-507. [CrossRef] [PubMed]

22. Bialonska, D.; Kasimsetty, S.G.; Schrader, K.K.; Ferreira, D. The effect of pomegranate (Punica granatum L.) byproducts and ellagitannins on the growth of human gut bacteria. J. Agric. Food Chem. 2014, 57, 8344-8349. [CrossRef] [PubMed]

23. Yang, H.; Hewes, D.; Salaheen, S.; Federman, C. Effects if blackberry juice on growth inhibition of foodborne pathogens and growth promotion of Lactobacillus. Food Control. 2014, 37, 15-20. [CrossRef]

24. Boutrou, R.; Guéguen, M. Interest in Geotrichum candidum for cheese technology. Int. J. Food Microbiol. 2005, 102, 1-20. [CrossRef] [PubMed]

25. Osawa, R.; Kuroiso, K.; Goto, S.; Shimizu, A. Isolation of tannin-depending lactobacilli from humans and fermented food. Appl. Environ. Microbiol. 2000, 66, 3093-3097. [CrossRef] [PubMed]

26. Li, M.S.; Yao, K.; He, Q.; Jia, D.Y. Biodegradation of gallotannins and ellagitannins. J. Basic. Microbiol. 2006, 1 , 68-84.

27. Gatto, M.A.; Ippolito, A.; Linsalata, V.; Cascarano, N.A.; Nigro, F.; Vanadia, S.; di Venere, D. Activity of extracts from wild edible herbs against postharvest fungal diseases of fruit and vegetables. Postharvest Biol. Technol. 2011, 61, 72-82. [CrossRef]

28. Yuvamoto, P.D.; Said, S. Germination, duplication cycle and septum formationare altered by caffeine, caffeic acid and cinnamic acid in Aspergillus nidulans. Microbiology 2007, 76, 735-738. [CrossRef]

29. Kanaani, J.; Ginsburg, H. Effects of cinnamic acid derivatives on in vitro growth of Plasmodium falciparum and on the permeability of the membrane of malariainfected erythrocites. Antimicrob. Agents Chemother. 1992, 36, 1102-1108. [CrossRef] [PubMed]

30. Sójka, M.; Kołodziejczyk, K.; Milala, J.; Abadias, M.; Viñas, I.; Guyot, S.; Baron, A. Composition and properties of the polyphenolic extracts obtained from industrial plum pomaces. J. Funct. Foods 2015, 12, 168-178. [CrossRef]

31. Sójka, M.; Klimczak, E.; Macierzyński, J.; Kołodziejczyk, K. Nutrient and polyphenolic composition of industrial strawberry press cake. Eur. Food Res. Technol. 2013, 237, 995-1007. [CrossRef]

32. Yamaguchi, M.U.; Garcia, F.P.; Cortez, D.A.G; Ueda-Nakamura, T.; Filho, B.P.D.; Nakamura, C.V. Antifungal effects of ellagitannin isolated from leaves of Ocotea odorifera (Lauraceae). Atonie Van Leuw. 2011, 99, 507-514. [CrossRef] [PubMed]

33. Gleń, K.; Boligłowa, E. The effect of PRP SOL fertilizer on the dynamics of phytopathogenic and antagonistic fungi growth in vito. J. Res. Appl. Agric. Eng. 2011, 56, 97-101.

Sample Availability: Samples of the REP preparation are available from the authors.

(C) 2016 by the authors; licensee MDPI, Basel, Switzerland. This article is an open access article distributed under the terms and conditions of the Creative Commons Attribution (CC-BY) license (http:/ / creativecommons.org/licenses/by/4.0/). 